\title{
La iguana negra, un nuevo habitante en la UNAM
}

\author{
Edmundo Pérez-Ramos
}

\section{Resumen}

Por primera vez se registra la presencia de esta especie exótica en la Reserva Ecológica de San Ángel (REPSA). Dentro del campus Ciudad Universitaria de la Universidad Nacional Autónoma de México (UNAM), en la Ciudad de México se avistó una iguana negra o garrobo (Ctenosaura pectinata).

Palabras clave: especie exótica, Iguana, Ctenosaura pectinata, Ciudad de México, REPSA.

\section{BLACK SPINY TAILED IGUANA, A NEW INHABITANT IN UNAM}

\begin{abstract}
For the first time the presence of this exotic species is registered in the San Ángel Ecological Reserve (REPSA). Within the campus of the University City of the National Autonomous University of Mexico (UNAM), a black iguana (Ctenosaura pectinata) was sighted in Mexico City.
\end{abstract}

Key words:exotic species, black spiny-tailed Iguana, Ctenosaura pectinata, Mexico City, REPSA. 
Figura 5.

Ejemplar adulto, macho de Ctenosaura pectinata sobreviviendo en la Ciudad Universitaria, 25 marzo 2014, $14: 30$ h; $19^{\circ} 19^{\prime} 32^{\prime \prime} \mathrm{N} ; 99^{\circ} 10^{\prime}$ $32^{\prime \prime} \mathrm{W}, 2273 \mathrm{~m}$.

\section{¿Cómo y dónde?}

A partir del avistamiento de un individuo de especie exótica, en este caso conocida como iguana negra o garrobo, y con el uso de una guía especializada en iguanas (IRCF Iguana ID Guide, 2010) se logró identificar el espécimen como Ctenosaura pectinata (ver figura 4). Sin embargo, el género está en revisión taxonómica (Zarza et al. 2008 y 2016; Buckley et al. 2016), por lo que se recomienda tomar con reserva el nombre de la especie, hasta que se establezcan los posibles parentescos.

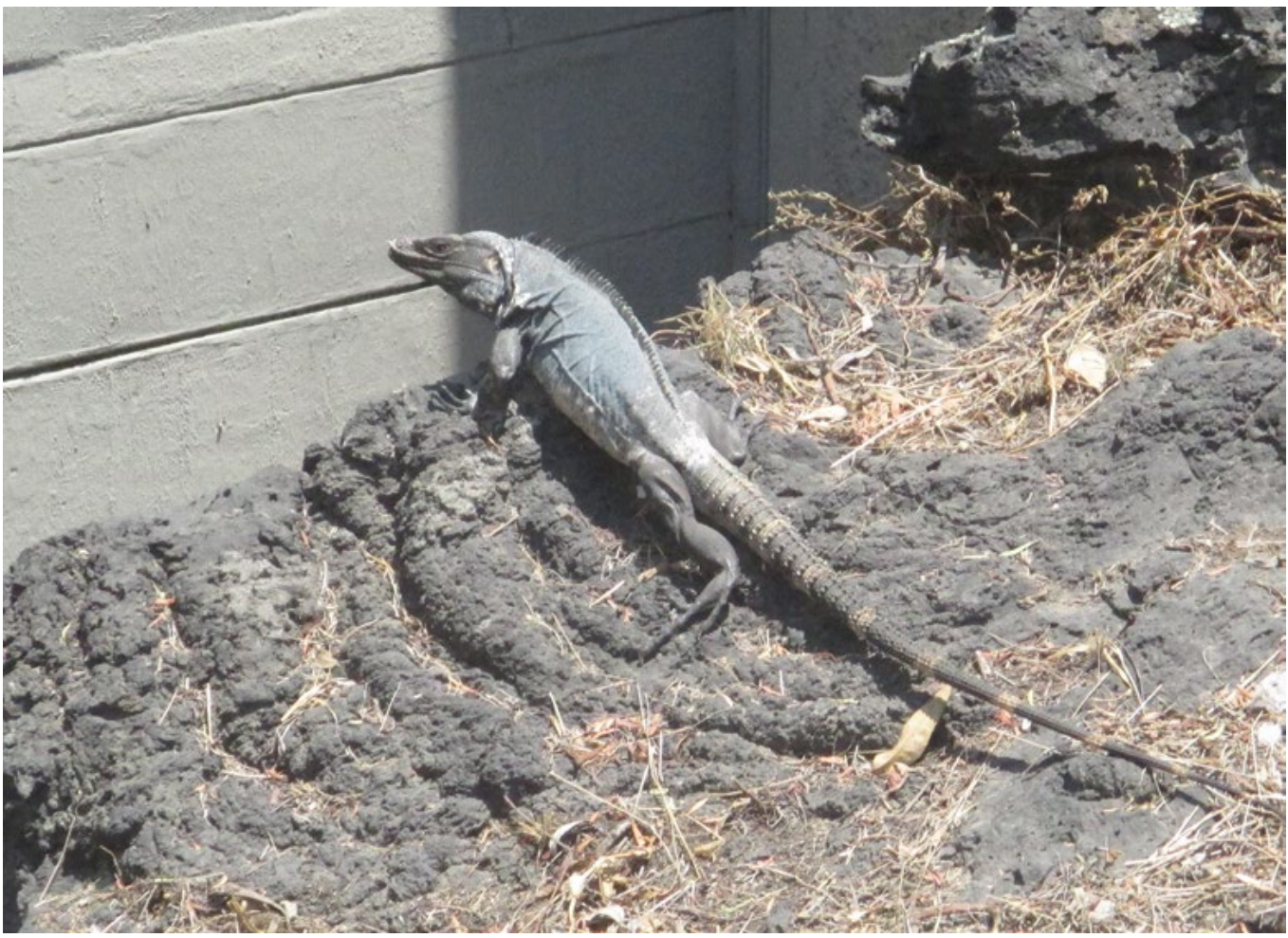

El ejemplar se observó dentro de las instalaciones de UNAM, en la esquina poniente del Instituto de Fisiología Celular, de la Ciudad Universitaria (190 19' 32" N; $99^{\circ}$ 10' 32" W; en una elevación de 2273 msnm), en un reducto del gran ecosistema de la REPSA, donde hasta el momento se han contabilizado más de 1500 especies nativas —entre plantas, animales, hongos, algas y microorganismos-, además de la mencionada serpiente exótica (Nava, 2015).

La especie C. pectinata es exclusiva de México, pero ha sido introducida en Estados Unidos (Zarza et al. 2008; Buckley et al. 2016). Naturalmente se distribuye en la zona mesoamericana de la vertiente del Pacífico y penetra tierra adentro, en la gran cuenca del Río Balsas que cubre varios estados al sur de la ciudad capital. Su hábitat se encuentra en zonas cálidas con altitudes desde bajas hasta intermedias, en climas cálidos y semicálidos. Puede extenderse desde el nivel 
del mar hasta 1800 metros sobre el nivel del mar (msnm), en áreas cubiertas por bosques tropicales caducifolios, espinosos y mixtos, o matorral xerófilo (plantas adaptadas a la escasez de agua), y subperennifolios (bosque tropical); aunque algunos ejemplares se han visto en bosques de encinos, mixtos de pino-encino y algunas zonas boscosas de pinos que descienden hasta 800 msnm y otros cerca del nivel del mar.

La iguana observada, es un ejemplar macho adulto que aproximadamente tiene una longitud total de un metro, fue visto y fotografiado al deambular sobre los pedregales en la temporada de sequía el viernes 15 de abril del 2016, aproximadamente a las 14:30 horas, exploraba un área rocosa de $30 \mathrm{~m}^{2}$ (jardín artificial), tal vez en busca de alimento. Por sus movimientos conductuales, al parecer saboreaba el ambiente al sacar y meter la viscosa y húmeda lengua constantemente, momentos después consumía flores de buganvilla. Aparentemente, este individuo como especie introducida, no nativa, se escapó o la dejaron en libertad, pero se desconoce su origen.

Figura 6.

Iguana negra deambulando en las inmediaciones del Instituto de Fisiología Celular, UNAM.
El hábitat de Ciudad Universitaria y de la REPSA es considerado como matorral xerófilo (como se observa en la figura 3), muy parecido a los que naturalmente habita este iguánido, sin embargo, el hábitat no cuenta con las especies de plantas que normalmente consume la iguana. Esta especie no ha logrado ascender los valles o cimas de las montañas del Valle por los requerimientos geográficos, además, se debe destacar que la dispersión del ser humano es un factor de consideración.

Se tiene la hipótesis de que la permanencia de esta iguana negra o garrobo en la megalópolis va a ser temporal, debido principalmente a características biológicas y requerimientos ecológicos; sin embargo, podría acondicionarse bien a una estancia en cautiverio donde, en teoría, podría reproducirse, ya que es ovípara, 
Cuija besucona Hemidactylus frenatus.

Autor: Miguel Ángel Sicilia Manzo, CONABIO
Serpiente pulsera o ciega de la especie Indotyphlops braminus. Autor: David V Raju, Wikipedia.org pero carece de un consorte y el clima de la CDMX (templado, de cálido a frío) no concuerda con los requerimientos ecológicos de reproducción (climas cálidos, semicálidos y secos).

En cambio, otras dos especies han sacado ventaja de este ciclo cálido en la ciudad y al nivel global, una es la lagartija cuija o besucona de la especie Hemidactylus frenatus, y otra es la serpiente pulsera o ciega de la especie Indotyphlops braminus. Ambas especies, actualmente cosmo-

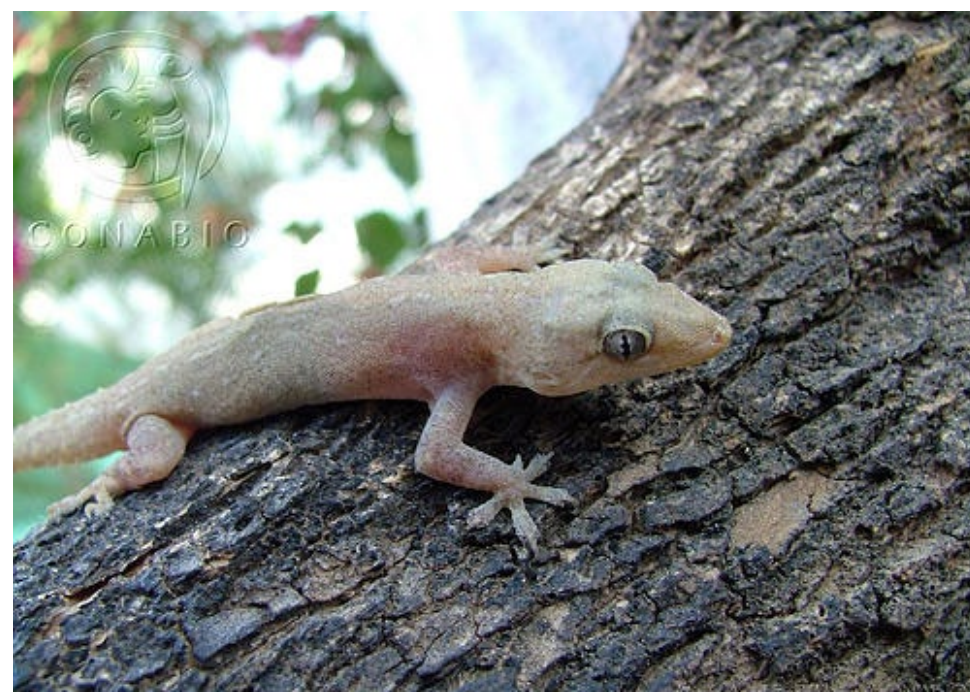
politas y abundantes, son ovíparas y cuentan con ventajas evolutivas que les han permitido vivir en ambientes aparentemente inhabitables. Cabe mencionar que la serpiente ciega se puede reproducir por si misma (partenogenética).

\section{¿Qué pasará con la iguana?}

Este gran saurio se ha logrado mantener en cautiverio como mascota domesticada, por lo tanto, cabe la posibilidad de que, al ser monitoreada y conseguírsele una pareja, pueda continuar viviendo en la REPSA e incluso reproducirse, pasando a ser residente perma-

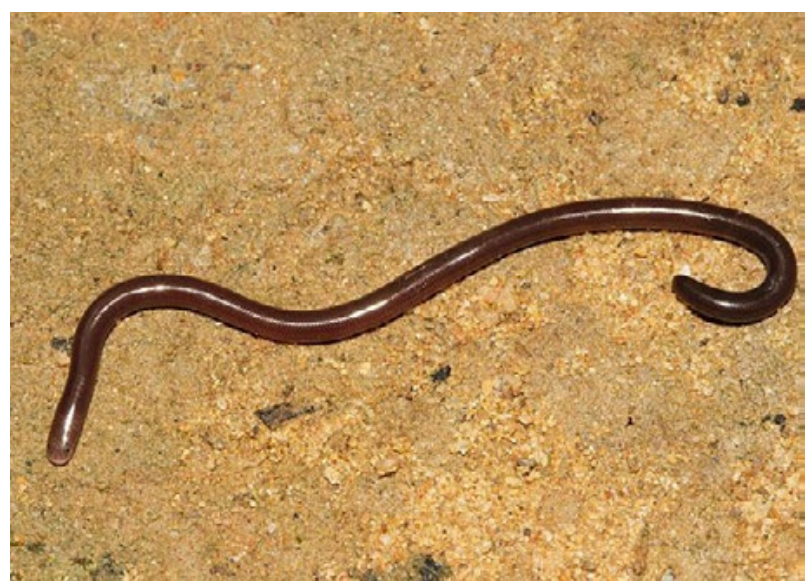
nente. Incluso, si hay necesidad, se podrían incubar sus huevos, como en las granjas para iguanas que existen en varios estados del país. Este espécimen se puede adaptar al clima de la CDMX mientras dure el ciclo sumamente cálido que vivimos a nivel mundial.

Sin embargo, el ecólogo Zenón Cano Santana (en comunicación personal), sugiere que se extirpe del área de la REPSA, debido principalmente a la experiencia que se tiene sobre los estragos ecológicos que han provocado las especies invasoras, entre ellas este iguánido en varias partes de América (Zarza et al. 2008, 2016; Buckley et al. 2016). 


\section{Estado de conservación}

Todas las especies de anfibios y reptiles son importantes biológicamente hablando, pero existen diversos reglamentos y normas que nos indican las especies que están consideradas en alguna categoría de conservación. En el caso de la iguana negra, la International Union for Conservation of Nature (IUCN) la reconoce como especie amenazada-vulnerable; la Norma Oficial Mexicana (NOM-59-SEMARNAT-2010) la cataloga como especie en riesgo; mientras que The Convention on International Trade in Endangered Species of Wild Fauna \& Flora (CITES) la ubica en la categoría de prioridad con manejo especial.

Tomando en cuenta todos estos criterios, se puede apuntar la importancia de la conservación de la especie Ctenosaura pectinata, tanto en su hábitat natural como en lugares alterados o en cautiverio, e incluso en lugares aparentemente inhóspitos.

\section{Agradecimientos}

A Hilda Marcela Pérez Escobedo, Guillermo Gil, Azucena Pérez Saldaña, Carina Itzel Gálvez García y a los revisores anónimos que apoyaron en diferentes etapas del estudio y fueron esenciales para mejor sustancialmente la publicación.

\section{Referencias}

* Buckley, L. J., De Queiroz, K., Grant, T. D., Hollingsworth, B. D., Iverson, J. B., Pasachnik, S. A. y Stephen, C. L. (2016). A Checklist of the Iguanas of the world (Iguanidae: Iguaninae). Herpetological Conservation \& Biology. 11 (Monograph 6. Iguanas: Biology, Systematics, and Conservation), 4-46.

* International Union for Conservation of Nature (IUCN). https://www.iucn.org/es.

* Méndez de la Cruz, F. R., Díaz de la Vega-Pérez, A. H. y Jiménez-Arcos, V. H. (2009). Biodiversidad del ecosistema del Pedregal de San Ángel. En Lot, A. y Cano- Santana, Z. (eds.). Biodiversidad del ecosistema del Pedregal de San Ángel. UNAM.

* Método de Evaluación Rápida de Invasividad (MERI) para especies exóticas en México Hemidactylus frenatus Schlegel, 1836. Recuperado de https://www.gob.mx/ cms/uploads/attachment/file/222326/Hemidactylus frenatus A.pdf

- Nava-G. C. A. (2015). Conoce la REPSA [tríptico]. Reserva Ecológica del Pedregal de San Ángel de la Ciudad Universitaria, Universidad Nacional Autónoma de México Recuperado de http://www.repsa.unam.mx/index.php/objetivosrepsa/divulgacion/productos. 
* Norma Oficial Mexicana NOM-59-ECOL-2010. SEMARNAT-2010. Secretaría de Medio Ambiente y Recursos Naturales, México, 30 de diciembre de 2010. Recuperado de http://www.semarnat.gob.mx.

* Raju, David V. (2013). Worm Snake [imagen]. Wikipedia.org. Recuperado de https:// en.wikipedia.org/wiki/Indotyphlops braminus\#/media/File:Davidraju Worm Snake.jpg.

* Ramírez-Bautista, A., Hernández-Salinas, U., García-Vázquez, U. O., Leyte-Manrique, A. y Canseco-Márquez, L. (2009). Herpetofauna del Valle de México: Diversidad y Conservación. Universidad Autónoma del Estado de Hidalgo y Comisión Nacional para el Conocimiento y uso de la Biodiversidad. México. 213 pp.

* Sicilia Manzo, Miguel Ángel (2012). Cuija besucona Hemidactylus frenatus [imagen]. CONABIO. Recuperado de /fotoweb/archives/5001-Reptiles/Animales/Vertebrados/Reptiles/MASM06860\%20Hemidactylus\%20frenatus.jpg.info.

- Stephen, Catherine (ed.) (2010). Ctenosaura Identification Guide. Utah Valley University, IRCF Iguana ID Guide. Recuperado de www.caftadr-environment.org/wpcontent/uploads/2016/04/IRCF-Iguana-ID-Guide-Spanish.pdf.

* The Convention on International Trade in Endangered Species of Wild Fauna and Flora (CITES).

* Uribe-Peña, Z., Ramírez-Bautista, A. y Casas-Andreu, G. (1999). Anfibios y Reptiles de las serranías del Distrito Federal, México. Universidad Nacional Autónoma de México, Instituto de Biología. Cuadernos 32. 119 pp.

* Zarza, E., Reynoso, V. H., y Emerson, B. C. (2016). Genetic Tools for assisting sustainable Management and conservation of the Spiny-Tailed Iguana, Ctenosaura pectinate [Supplementary Materials]. Herpetological Conservation \& Biology. 11(Monograph 6, Iguanas: Biology, Systematics, and Conservation): 255-264.

* Zarza, E., Reynoso, V. H., y Emerson, B. C. (2008). Diversification in the north neotropics: mitocondrial and nuclear DNA phylogeography of the iguana Ctenosaura pectinata and related species. Molecular Ecology. 17: 3259-3275.

\section{Cómo citar este artículo}

* Pérez-Ramos, Edmundo (2018). La iguana negra, un nuevo habitante en la UNAM. Revista Digital Universitaria (RDU). Vol. 19, núm. 5 septiembre-octubre. DOI: http:// doi.org/10.22201/codeic.16076079e.2018.v19n5.a9. 